\title{
杂化钙钛矿 $\left(\mathrm{HOC}_{2} \mathrm{H}_{4} \mathrm{NH}_{3}\right)_{2} \mathrm{CuCl}_{4}$ 的制备与表征
}

\author{
杨志胜 ${ }^{1}$ ，柯蔚芳 ${ }^{2}$, 王艳香 ${ }^{1}$ ，黄丽群 ${ }^{1}$, 郭平春 ${ }^{1}$ ，朱 华 ${ }^{3}$ \\ (景德镇陶瓷大学 1. 材料科学与工程学院; 2. 陶瓷美术学院; 3. 机电学院, 景德镇 333403)
}

摘 要: 采用低温溶液法合成了新型层状有序的含有羟基的有机/无机杂化钻钠矿材料 $\left(\mathrm{HOC}_{2} \mathrm{H}_{4} \mathrm{NH}_{3}\right)_{2} \mathrm{CuCl}_{4}$, 采用元 素分析、红外光谱、紫外-可见光吸收光谱、 $\mathrm{X}$ 射线衍射和 X 射线吸收精细结构等手段对其结构与性能进行了表征。 结果表明: 该材料通过无机框架诱导有机组分有序排列, 形成了规则的层状结构, 有序性高。该杂化钙钛矿材料的 分解温度为 $212^{\circ} \mathrm{C}$, 电阻率为 $2.86 \times 10^{6} \Omega \cdot \mathrm{cm}$, 比不含羟基杂化钻钛矿的电阻率低两个数量级。紫外-可见光吸收光 谱显示 $285 \mathrm{~nm}$ 左右有一归因于电子从 $\mathrm{Cl}(3 \mathrm{p})$ 价带顶跃迁到 $\mathrm{Cu}(4 \mathrm{~s})$ 导带底而产生的吸收峰。X 射线吸收精细结构谱 图表明: 二维层状杂化钻铁矿晶体中的 $\mathrm{Cu}^{2+}$ 与 6 个 $\mathrm{Cl}^{-}$形成八面体配位, $\mathrm{Cu}-\mathrm{Cl}$ 键长为 $0.191 \mathrm{~nm}$, 层间距为 $1.099 \mathrm{~nm}$ 。 关 键 词: 有机/无机杂化; 钻钛矿; 晶体结构

中图分类号: O614 文献标识码: A

\section{Preparation and Characterization of a Novel Hybrid Perovskite $\left(\mathrm{HOC}_{2} \mathrm{H}_{4} \mathrm{NH}_{3}\right)_{2} \mathrm{CuCl}_{4}$}

\author{
YANG Zhi-Sheng ${ }^{1}$, KE Wei-Fang ${ }^{2}$, WANG Yan-Xiang ${ }^{1}$, HUANG Li-Qun ${ }^{1}$, GUO Ping-Chun ${ }^{1}$, ZHU Hua ${ }^{3}$ \\ (1. Department of Materials Science and Engineering, Jingdezhen Ceramic Institute, Jingdezhen 333403, China; 2. Department \\ of Ceramic Art, Jingdezhen Ceramic Institute, Jingdezhen 333403, China; 3. Department of Mechanical and Electrical Engi- \\ neering, Jingdezhen Ceramic Institute, Jingdezhen 333403, China)
}

\begin{abstract}
A novel organic-inorganic hybrid material containing hydroxyl, $\left(\mathrm{HOC}_{2} \mathrm{H}_{4} \mathrm{NH}_{3}\right)_{2} \mathrm{CuCl}_{4}$ with perovskite structure, was synthesized by low-temperature solution method. The structure and properties of the hybrid material were characterized by elemental analysis, IR, UV-Vis absorption spectrum, X-ray diffraction, and X-ray absorption fine structure techniques. The result reveals that the hybrid material $\left(\mathrm{HOC}_{2} \mathrm{H}_{4} \mathrm{NH}_{3}\right)_{2} \mathrm{CuCl}_{4}$ is well-ordered and in two-dimensional layered frame, and the organic component is in orderly arrangement induced by inorganic framework. The decomposition temperature of the hybrid material is about $212^{\circ} \mathrm{C}$, and the resistance of the hybrid material is about $2.86 \times 10^{6} \Omega \cdot \mathrm{cm}$, which is two orders of magnitude less than hybrid perovskite without hydroxyl. UV-Vis spectra shows that the crystal has a new absorption peak at about $285 \mathrm{~nm}$ which is attributed to the electronic transitions from the top of the valence band of $\mathrm{Cl}(3 \mathrm{p})$ to the bottom of the conduction band of $\mathrm{Cu}(4 \mathrm{~s})$. XAFS result shows that $\mathrm{Cu}^{2+}$ with six $\mathrm{Cl}^{-}$forms octahedral coordination in the hybrid layered crystal with layer spacing of $1.099 \mathrm{~nm}$, and the $\mathrm{Cu}-\mathrm{Cl}$ bond length is about $0.191 \mathrm{~nm}$.
\end{abstract}

Key words: organic-inorganic hybrid; perovskite; crystal structure

收稿日期: 2016-12-27; 收到修改稿日期：2017-02-14

基金项目: 国家自然科学基金(51403090，51462015); 江西省教育厅青年自然科学基金(GJJ150897); 景德镇陶瓷大学博士 科研启动项目(2010); 江西省高等学校大学生创新创业计划项目(2017)

National Natural Science Foundation of China(51403090, 51462015); Youth Natural Science Foundation of Education Department of Jiangxi Province (GJJ150897); Doctoral Research Project of Jingdezhen Ceramic Institute(2010); Innovation and Entrepreneurship Program of College Students in Jiangxi Province (2017)

作者简介: 杨志胜(1981-), 男, 博士, 讲师. E-mail: 85694626@qq.com 
二维层状有机/无机杂化䥻钛矿将有机和无机 半导体材料的优点结合在一个分子复合物内, 可以 结合二者的优势, 弥补各自的不足, 其光、电、磁和 热力学等性质引起了广泛关注 ${ }^{[1-3]}$ 。杂化钙钛矿具有 优异的半导体性质, 在电致发光器件、场效应晶体 管和太阳电池器件上的研究成为当前杂化钙钣矿材 料研究的主要方向 ${ }^{[4-6]}$ 。通过选择不同的有机和无机 组分, 可以改变钙钛矿的晶体结构, 从而调节其禁 带宽度 ${ }^{[7-8]}$ 。但基于二维层状结构的杂化钲钣矿材料 的电阻率较大, 又限制了其在光电领域的应用。为 了降低二维层状杂化钙钛矿的电阻率, 本工作选用 含有羟基的乙醇胺盐酸盐与氯化铜合成了 $\left(\mathrm{HOC}_{2} \mathrm{H}_{4} \mathrm{NH}_{3}\right)_{2} \mathrm{CuCl}_{4}$, 采用元素分析、 $\mathrm{X}$ 射线衍射和 红外光谱等多种测试手段, 表征产物的组成和结构, 并初步研究了其性质。

\section{1 实验方法}

\section{1 化学试剂}

实验所用的乙醇胺、盐酸、无水乙醇、 $\mathrm{CuCl}_{2}$ 、 乙醚等化学试剂均购自阿拉丁试剂有限公司。

\section{2 样品的制备}

在冰水浴下，向乙醇胺的无水乙醇溶液中缓慢 滴加适量的浓盐酸, 充分摚拌后加热制备铵盐使物 质的量之比为 $1: 2$ 的 $\mathrm{CuCl}_{2}$ 的无水乙醇溶液与 $\mathrm{HOC}_{2} \mathrm{H}_{4} \mathrm{NH}_{3} \mathrm{Cl}$ 的无水乙醇溶液混合, $80^{\circ} \mathrm{C}$ 下加热冷 凝回流 $1 \mathrm{~h}$ 缓慢降温至室温析出晶体, 反应产物经 过滤、乙醚反复冲洗数次, 得到滤饼, 滤饼在乙醇中 重结晶, 得到产物 $\left(\mathrm{HOC}_{2} \mathrm{H}_{4} \mathrm{NH}_{3}\right)_{2} \mathrm{CuCl}_{4}$ 晶体, 置于 $60^{\circ} \mathrm{C}$ 真空烘箱中干燥 $24 \mathrm{~h}$, 保存在干燥器中。产物 元素分析(Perkin- Elmer 240C 元素分析仪, 美国)结 果为 (括号内为理论值): $\mathrm{C} 14.26 \%(14.58 \%), \mathrm{N}$ $8.23 \%$ (8.50\%), H 4.94\% (4.89\%)。

将 $\left(\mathrm{HOC}_{2} \mathrm{H}_{4} \mathrm{NH}_{3}\right)_{2} \mathrm{CuCl}_{4}$ 晶体配成 $40 \mathrm{mg} / \mathrm{mL}$ 的 无水乙醇溶液, 用台式匀胶机( KW-4A 型, 中国科 学院微电子研究室) 以 $2000 \mathrm{r} / \mathrm{min}$ 的速率旋转涂覆 $30 \mathrm{~s}$, 在石英祄底上得到较为平整的薄膜。

\section{3 结构表征}

在德国 Brucker 公司 VII 型傅里叶变换红外光 谱测试仪上测试样品的红外光谱(FT-IR), 采用 $\mathrm{KBr}$ 压片。在美国 PE 公司生产的 Lambda20 紫外-可见 光谱仪上测试样品的紫外-可见吸收光谱。采用日本 理学公司 D/max-r 型衍射仪测试样品的 X 射线衍射 图谱(XRD)。采用 Van der Pauw 法在美国 BIO-Rad microscience HL5500 Hall System 测量样品的电阻 率。采用美国 SDT 公司 Q600 型热分析仪进行热分
析(TGA)。采用 Huber 多圆衍射仪进行 X 射线吸收 精细结构(XAFS)分析, 样品前狭缝为 $1 \mathrm{~mm} \times 1 \mathrm{~mm}$, 两个后狭缝垂直方向分别为 $2 \mathrm{~mm}$ 和 $1 \mathrm{~mm}, X$ 射线 波长为 $0.129 \mathrm{~nm}$ 。

\section{2 结果与讨论}

\section{1 红外光谱(FT-IR)分析}

从 $\left(\mathrm{HOC}_{2} \mathrm{H}_{4} \mathrm{NH}_{3}\right)_{2} \mathrm{CuCl}_{4}$ 的红外光谱图(图 1)中 可以看出, $\left(\mathrm{HOC}_{2} \mathrm{H}_{4} \mathrm{NH}_{3}\right)_{2} \mathrm{CuCl}_{4}$ 在 $3123 \mathrm{~cm}^{-1}$ 出现一 个新的宽峰。此频率值比相应的乙醇胺的 $v(\mathrm{NH})$ 低, 而又比相应的乙醇胺盐酸盐的 $v(\mathrm{NH})$ 高, 说明 $\left(\mathrm{HOC}_{2} \mathrm{H}_{4} \mathrm{NH}_{3}\right)_{2} \mathrm{CuCl}_{4}$ 中形成了较强的 $\mathrm{N}-\mathrm{H} \cdots \cdots \mathrm{Cl}$ 氢键, 并且此氢键的强度比相应的烷基胺盐的要 弱。在 $\left(\mathrm{HOC}_{2} \mathrm{H}_{4} \mathrm{NH}_{3}\right)_{2} \mathrm{CuCl}_{4}$ 中, 每一个 $\mathrm{NH}_{3}{ }^{+}$基团与 三个 $\mathrm{Cl}^{-}$形成氢键, 而 $\mathrm{Cl}^{-}$与无机层中的 $\mathrm{Cu}^{2+}$ 形成配 位键 ${ }^{[9]}$ 。

\section{2 紫外-可见光吸收光谱(UV-Vis)分析}

从石英基底上的 $\left(\mathrm{HOC}_{2} \mathrm{H}_{4} \mathrm{NH}_{3}\right)_{2} \mathrm{CuCl}_{4}$ 旋涂薄膜 的紫外-可见光吸收光谱图(图 2)中可以看到, $285 \mathrm{~nm}$ 处有一吸收峰, 单纯的有机组分和无机组分都没有 紫外吸收，这说明在有机和无机组分之间形成了新 的杂化钲钛矿结构。在二维无机层中的光生激子产 生了吸收峰, $285 \mathrm{~nm}$ 左右的吸收峰归因于电子从 $\mathrm{Cl}(3 \mathrm{p})$ 价带顶跃迁到 $\mathrm{Cu}(4 \mathrm{~s})$ 导带底 ${ }^{[10]}$ 。

\section{$2.3 \mathrm{X}$ 射线衍射分析}

图 3 为 $\left(\mathrm{HOC}_{2} \mathrm{H}_{4} \mathrm{NH}_{3}\right)_{2} \mathrm{CuCl}_{4}$ 粉末的 XRD 图谱, 从图中可以看出, 粉末衍射峰非常强, 主要为等间 距的垂直于 $\mathrm{c}$ 轴的 $(00 l, l=2,4,6,8 \cdots \cdots)$ 面的衍射 峰, 说明产物结晶性能很好, 并且产物具有二维层 状结构。根据布拉格公式:

$$
2 d \sin \theta=n \lambda
$$

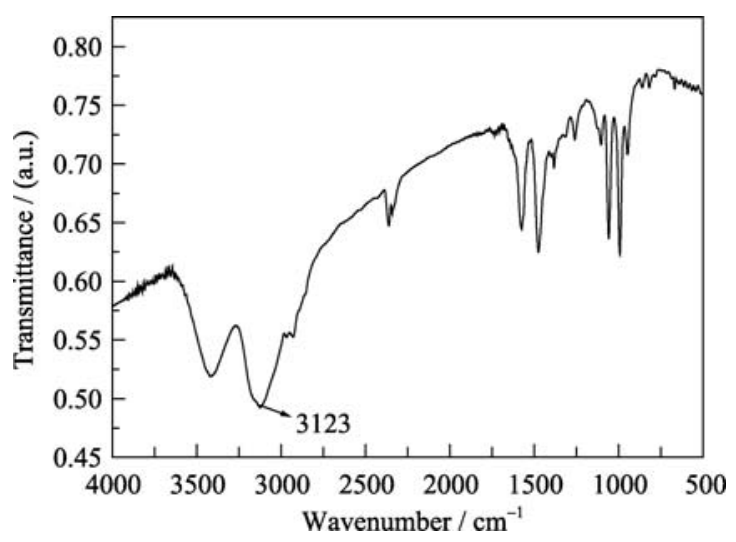

图 $1\left(\mathrm{HOC}_{2} \mathrm{H}_{4} \mathrm{NH}_{3}\right)_{2} \mathrm{CuCl}_{4}$ 的红外光谱图

Fig. 1 FT-IR spectrum of $\left(\mathrm{HOC}_{2} \mathrm{H}_{4} \mathrm{NH}_{3}\right)_{2} \mathrm{CuCl}_{4}$ 


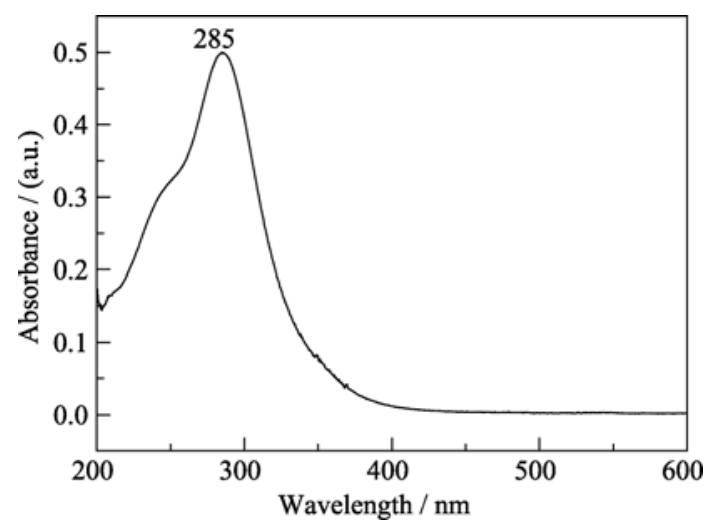

图 $2\left(\mathrm{HOC}_{2} \mathrm{H}_{4} \mathrm{NH}_{3}\right)_{2} \mathrm{CuCl}_{4}$ 的紫外-可见光光谱图

Fig. 2 UV-Vis spectrum of $\left(\mathrm{HOC}_{2} \mathrm{H}_{4} \mathrm{NH}_{3}\right)_{2} \mathrm{CuCl}_{4}$

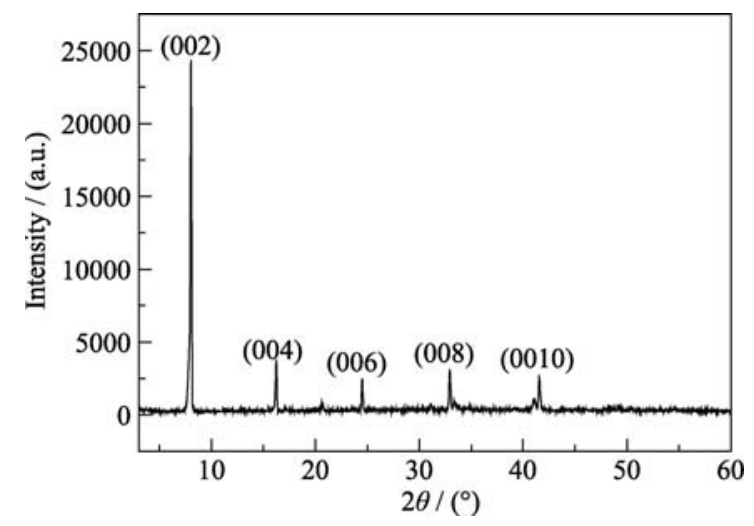

图 $3\left(\mathrm{HOC}_{2} \mathrm{H}_{4} \mathrm{NH}_{3}\right)_{2} \mathrm{CuCl}_{4}$ 粉末的 $\mathrm{XRD}$ 图谱

Fig. 3 XRD pattern of $\left(\mathrm{HOC}_{2} \mathrm{H}_{4} \mathrm{NH}_{3}\right)_{2} \mathrm{CuCl}_{4}$ powder

采用单色 $\mathrm{CuK} \alpha$ 辐射, $\lambda$ 为 $0.1540562 \mathrm{~nm}$, 当 $n$ 等于 1 时, 从 XRD 在小角范围内的 (002) 面上的第一 级衍射峰 $\left(2 \theta=8.04^{\circ}\right)$ 可以计算出层间距 $d$ 值为 $1.099 \mathrm{~nm}$ 。

根据文献报道，在直链烷基化合物中，烷基铵 链采取全反式构象，而且与无机层并不是垂直排列， 所有的有机链几乎具有同样的倾斜角 ${ }^{[11]}$ 。由此得到, 层状钙钛矿 $\left(\mathrm{HOC}_{2} \mathrm{H}_{4} \mathrm{NH}_{3}\right)_{2} \mathrm{CuCl}_{4}$ 中有机链的结构和 取向示意图见图 4 所示。

采用粉末压片法对 $\left(\mathrm{HOC}_{2} \mathrm{H}_{4} \mathrm{NH}_{3}\right)_{2} \mathrm{CuCl}_{4}$ 进行制 样, 然后测试其霍尔电阻率, 测得电阻率为 $2.86 \times$

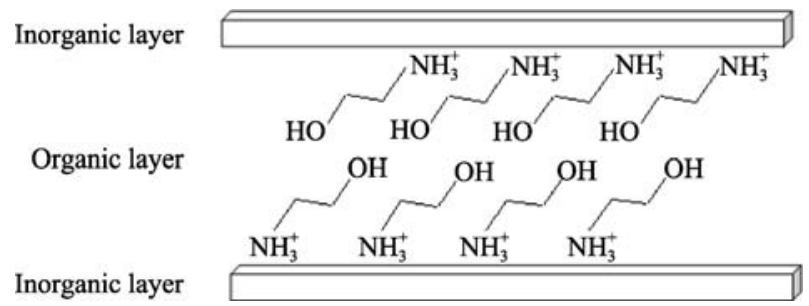

图 4 层状 $\left(\mathrm{HOC}_{2} \mathrm{H}_{4} \mathrm{NH}_{3}\right)_{2} \mathrm{CuCl}_{4}$ 中有机链的结构和取向示意图 Fig. 4 Schematic illustration of the structure and orientation in the layered $\left(\mathrm{HOC}_{2} \mathrm{H}_{4} \mathrm{NH}_{3}\right)_{2} \mathrm{CuCl}_{4}$ hybrid
$10^{6} \Omega \cdot \mathrm{cm}$ ，比文献报道的不含羟基的单铵盐 $\mathrm{CuCl}_{2}$ 基钻钣矿的数值低两个数量级 ${ }^{[12]}$ 。这是由于在单铵 盐钙铁矿中, 为了能适应无机层的空间, 有机胺链 段必须采取较大的扭曲，因而除了存在氢键外，有 机分子层间还存在较弱的范德华力, 导致有序性不 高。而在 $\left(\mathrm{HOC}_{2} \mathrm{H}_{4} \mathrm{NH}_{3}\right)_{2} \mathrm{CuCl}_{4}$ 中, 由于羟基的存在, 在两层有机分子层间主要存在的是较强的氢键, 排 列的有序性更高, 因而电阻率较小 ${ }^{[2]}$, 这有利于其 在光电材料领域的应用。

\section{$2.4 \mathrm{X}$ 射线吸收精细结构(XAFS)分析}

图 5 是杂化钻铁矿 $\left(\mathrm{HOC}_{2} \mathrm{H}_{4} \mathrm{NH}_{3}\right)_{2} \mathrm{CuCl}_{4}$ 中 $\mathrm{Cu}$ 的 $\mathrm{K}$ 边 XANES 谱。图 6 是 $\left(\mathrm{HOC}_{2} \mathrm{H}_{4} \mathrm{NH}_{3}\right)_{2} \mathrm{CuCl}_{4}$ 的 $\mathrm{Cu}$ 边傅里叶变换的 EXAFS 谱, 从图中可以看出, 在 $0.191 \mathrm{~nm}$ 处有一个源于 $\mathrm{Cu}-\mathrm{Cl}$ 的较强峰, 归属于 $\left(\mathrm{HOC}_{2} \mathrm{H}_{4} \mathrm{NH}_{3}\right)_{2} \mathrm{CuCl}_{4}$ 晶体第一近邻配位壳层中 $\mathrm{Cl}^{-}$ 的散射。通过一个 $\mathrm{Cu}-\mathrm{Cl}$ 八面体结构拟合发现，拟 合参数可以与实验谱吻合得很好 ${ }^{[13-14]}$, 拟合参数如 表 1 所示。从表 1 中可以看出, $\mathrm{Cu}^{2+}$ 和 $\mathrm{Cl}^{-}$形成 6 配

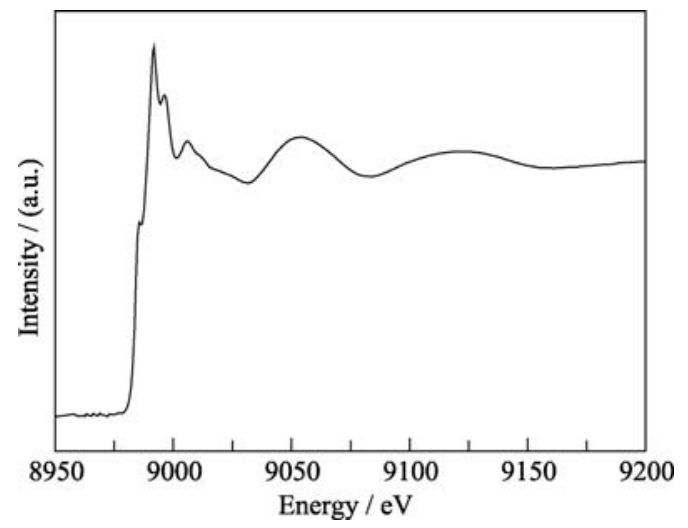

图 $5\left(\mathrm{HOC}_{2} \mathrm{H}_{4} \mathrm{NH}_{3}\right)_{2} \mathrm{CuCl}_{4}$ 的 $\mathrm{Cu}$ 的 $\mathrm{K}$ 边 XANES 谱 Fig. 5 XANES spectrum of $\mathrm{Cu}$ K-edge in $\left(\mathrm{HOC}_{2} \mathrm{H}_{4} \mathrm{NH}_{3}\right)_{2}$ $\mathrm{CuCl}_{4}$

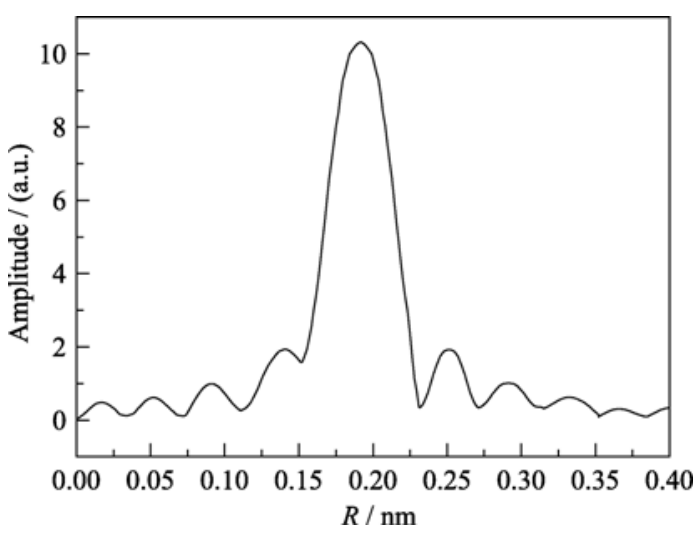

图 $6\left(\mathrm{HOC}_{2} \mathrm{H}_{4} \mathrm{NH}_{3}\right)_{2} \mathrm{CuCl}_{4}$ 的 $\mathrm{Cu}$ 边傅里叶变换的 EXAFS 谱 Fig. 6 Fourier transforms of EXAFS spectrum of $\mathrm{Cu}$ edge in $\left(\mathrm{HOC}_{2} \mathrm{H}_{4} \mathrm{NH}_{3}\right)_{2} \mathrm{CuCl}_{4}$ 
表 $1\left(\mathrm{HOC}_{2} \mathrm{H}_{4} \mathrm{NH}_{3}\right)_{2} \mathrm{CuCl}_{4}$ 的 EXAFS 拟合参数 Table 1 EXAFS fitting parameters of $\left(\mathrm{HOC}_{2} \mathrm{H}_{4} \mathrm{NH}_{3}\right)_{2} \mathrm{CuCl}_{4}$

\begin{tabular}{ccccc}
\hline Sample & Bond & $\mathrm{N}$ & $R / \mathrm{nm}$ & $\sigma^{2} / \mathrm{nm}^{2}$ \\
\hline$\left(\mathrm{HOC}_{2} \mathrm{H}_{4} \mathrm{NH}_{3}\right)_{2} \mathrm{CuCl}_{4}$ & $\mathrm{Cu}-\mathrm{Cl}$ & $6.0 \pm 0.5$ & $0.191 \pm 0.001$ & $4.8 \times 10^{-5}$ \\
\hline
\end{tabular}

位的八面体结构; $\sigma^{2}$ 为无序度因子, 包括结构无序 和热无序, 该材料的无序度较小, 说明其内部排列 规整, 有序度较高。根据 $\left(\mathrm{HOC}_{2} \mathrm{H}_{4} \mathrm{NH}_{3}\right)_{2} \mathrm{CuCl}_{4}$ 的层 间距和 $\mathrm{Cu}-\mathrm{Cl}$ 八面体结构参数, 得到层状钙铁矿的 结构示意图如图 7 所示。

\section{5 热分析}

样品 $\left(\mathrm{HOC}_{2} \mathrm{H}_{4} \mathrm{NH}_{3}\right)_{2} \mathrm{CuCl}_{4}$ 的 TGA 曲线如图 8 所 示 (温度范围: $20 \sim 600^{\circ} \mathrm{C}, \mathrm{N}_{2}$ 保护, $10^{\circ} \mathrm{C} / \mathrm{min}$ 的升温 速率), 从 TGA 曲线可以看出, 样品的分解温度为 $212.6^{\circ} \mathrm{C}$ 。这是由于在有机分子中含有羟基, 出现了 可逆的热力学结构转变 ${ }^{[14]}$ 。从 DSC 曲线上可以看出, 样品在 $100^{\circ} \mathrm{C}$ 以下有一个较宽的吸热峰, 对应于样 品的固-固相转变, 这可能与晶体中引入羟基形成 的分子间氢键的破坏相关。

热重分析说明在较宽的温度范围内材料一直都 保持结晶有序结构，稳定性较好，用于制备器件时

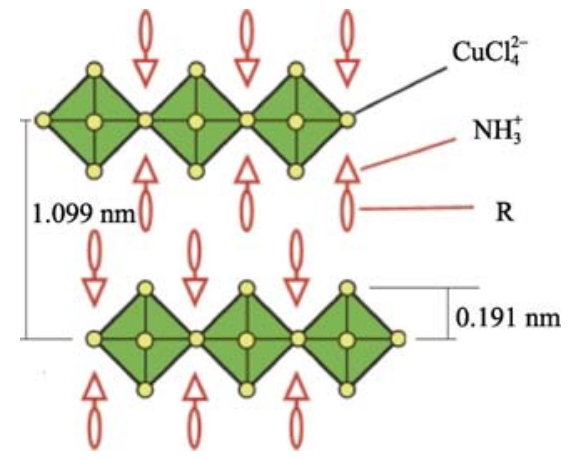

图 $7 \quad\left(\mathrm{HOC}_{2} \mathrm{H}_{4} \mathrm{NH}_{3}\right)_{2} \mathrm{CuCl}_{4}$ 晶体(100)面示意图

Fig. 7 Schematic representation of single-layer (100)-oriented $\left(\mathrm{HOC}_{2} \mathrm{H}_{4} \mathrm{NH}_{3}\right)_{2} \mathrm{CuCl}_{4}$ perovskites

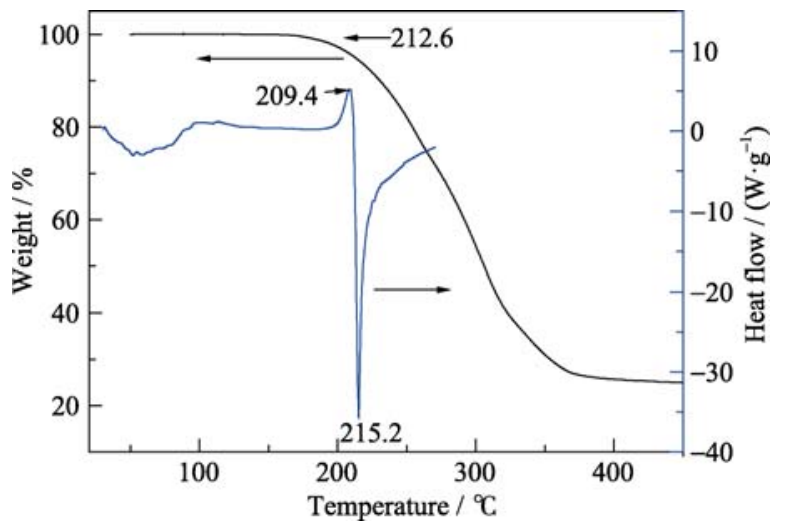

图 $8\left(\mathrm{HOC}_{2} \mathrm{H}_{4} \mathrm{NH}_{3}\right)_{2} \mathrm{CuCl}_{4}$ 的 TGA 和 DSC 曲线

Fig. 8 TGA and DSC curves of $\left(\mathrm{HOC}_{2} \mathrm{H}_{4} \mathrm{NH}_{3}\right)_{2} \mathrm{CuCl}_{4}$
有望在较广的使用范围获得高载流子迁移率。

\section{3 结论}

使用简单、低成本的溶液法成功获得具有二维 层状钙钛矿结构的有机/无机杂化晶体材料 $\left(\mathrm{HOC}_{2} \mathrm{H}_{4} \mathrm{NH}_{3}\right)_{2} \mathrm{CuCl}_{4}$, 该材料具有规则的层状结构, 层间距为 $1.099 \mathrm{~nm}$, 分解温度达到 $212^{\circ} \mathrm{C}$ 。通过 $\mathrm{X}$ 射线吸收精细结构分析证实了 $\left(\mathrm{HOC}_{2} \mathrm{H}_{4} \mathrm{NH}_{3}\right)_{2} \mathrm{CuCl}_{4}$ 钙钛矿材料中 $\mathrm{Cu}^{2+}$ 与 6 个 $\mathrm{Cl}^{-}$形成键长为 $0.191 \mathrm{~nm}$ 的配位。该材料在 $285 \mathrm{~nm}$ 左右出现新的吸收峰, 显 示了其在紫外探测上广阔的应用前景。羟基的引入 使得钻钛矿晶体中有机分子层间主要存在的是较强 的氢键，排列的有序性更高，从而具有较低的电阻 率和较高的稳定性。研究结果为今后钙钛矿材料的 设计提供了新的依据和方向。

致谢: 感谢中国科技大学同步辐射实验室提供 X 射 线吸收精细结构(XAFS)的测试与分析。

\section{参考文献:}

[1] MITZI D B. Synthesis, structure, and properties of organic -inorganic perovskites and related materials. Progress in Inorganic Chemistry, 1999, 48: 1-121.

[2] MITZI D B, CHONDROUDIS K, KAGAN C R. Organicinorganic electronics. IBM Journal of Research and Development, 2001, 45(1): 29-45.

[3] KAGAN C R, MITZI D B, DIMITRAKOPOULOS C D. Organicinorganic hybrid materials as semiconducting channels in thin-film field-effect transistors. Science, 1999, 286(5441): 945-947.

[4] WANG YAN-XIANG, LUO JUN, GUO PING-CHUN, et al. Application and development of hybrid perovskite materials in the field of solar cells. Journal of Inorganic Materials, 2015, 30(7): 673-682.

[5] SALIBA M, MATSUI T, DOMANSKI K, et al. Incorporation of rubidium cations into perovskite solar cells improves photovoltaic performance. Science, 2016, 354(6309): 206-209.

[6] XU Z T, MITZI D B, DIMITRAKOPOULOS C D, et al. Semiconducting perovskites $\left(2-\mathrm{XC}_{6} \mathrm{H}_{4} \mathrm{C}_{2} \mathrm{H}_{4} \mathrm{NH}_{3}\right)_{2} \mathrm{SnI}_{4}(\mathrm{X}=\mathrm{F}, \mathrm{Cl}$, $\mathrm{Br})$ : steric interaction between the organic and inorganic layers. Inorganic Chemistry, 2003, 42(6): 2031-2039.

[7] MITZI D B, DIMITRAKOPOULOS C D, KOSBAR L L Structurally tailored organic-inorganic perovskites: optical properties and solution-processed channel materials for thin-film transistors. Chemistry of Materials, 2001, 13(10): 3728-3740.

[8] BI D Q, TRESS W G, DAR M I, et al. Efficient luminescent solar cells based on tailored mixed-cation perovskites. Sci. Adv., 2016, 2: 
$1-7$.

[9] CHENG Z Y, GAO B X, PANG M L, et al. Preparation and characterization of a novel layered perovskite-type organic -inorganic hybrid material containing silica networks. Chem. Mater, 2003, 15(25): 4705-4708.

[10] WANG W, CHEN X, EFRIMA S. Fabrication of semiconductor nanoparticles in a three-dimensional organic-layered solid crystal. Chem. Mater, 1999, 11(7): 1883-1889.

[11] VENKATARAMAN N V, BARMA S, VASUDEVAN S, et al. Structural analysis of alkyl chain conformation in the layered organic-inorganic hybrids $\left(\mathrm{C}_{n} \mathrm{H}_{2 n+1} \mathrm{NH}_{3}\right)_{2} \mathrm{PbI}_{4}(n=12,16,18)$ by IR spectroscopy. Chem. Phys. Lett., 2002, 358(1): 139-143.

[12] MITZI D B. Synthesis, crystal structure, and optical and thermal properties of $\left(\mathrm{C}_{4} \mathrm{H}_{9} \mathrm{NH}_{3}\right)_{2} \mathrm{MI}_{4}(\mathrm{M}=\mathrm{Ge}, \mathrm{Sn}, \mathrm{Pb})$. Chem. Mater., 1996, 8(3): 791-800.

[13] DAI B, WANG Q, MA J Y. XAFS study of $\mathrm{Cu}^{2+}$ in aqueous solution of $\mathrm{CuBr}_{2}$. Nuclear Science and Techniques, 2012, 23(3): 129-133.

[14] XIAO Z L, CHEN H Z, SHI M M, et al. Preparation and characterization of organic-inorganic hybrid perovskite $\left(\mathrm{C}_{4} \mathrm{H}_{9} \mathrm{NH}_{3}\right)_{2} \mathrm{CuCl}_{4}$. Materials Science and Engineering B, 2005, 117: 313-316. 